\title{
СИСТЕМА УПРАВЛЕНИЯ И МОДЕЛИРОВАНИЯ БЕСПИЛОТНОГО ЛЕТАТЕЛЬНОГО АППАРАТА РАRRОТ
}

\section{PARROT UNMANNED AREA VEHICLE CONTROL SYSTEM AND MODELING}

\section{A. Davtyan}

K. Melikyan

Summary. The mathematical model of an unmanned aerial vehicle (UAV) Parrot Ar. Drone 2 was considered. The UAV control system in the SIMULINK graphical environment of MATLAB software was developed using a mathematical model. To control the movement of the UAV was developed proportional-integral-derivative (PID) regulators. Presented the coefficients of PID regulators obtained through the research. The analysis of results of test experiments is presented.

Keywords: Control System, unmanned aerial vehicle, PID regulator, Parrot Ar. Drone, UAV.

\author{
Давтян Артур Вазгенович \\ Аспирант, Начиональный политехнический \\ университет Армении \\ arturdavtyan1995@gmail.com \\ Меликян Карен Артурович \\ Национальный политехнический университет \\ Армении \\ karenmeliqyan1996@gmail.com
}

Аннотация. Рассмотрена математическая модель беспилотного летательного аппарата (БПЛА) Parrot Ar. Drone 2. Была разработана система управления БПЛА в графической среде SIMULINK программного обеспечения MATLAB c помощью математической модели. Для управления движением БПЛА была разработана пропорционально-интегрально-дифференцирующий (ПИД) регуляторы. Представлены коэффициенты ПИД-регуляторов, полученные в результате исследования. Представлен анализ результатов тестовых экспериментов.

Ключевые слова: система управления, беспилотный летательный аппарат, ПИД-регулятор, БПЛА, Parrot Ar. Drone 2.0.

ления, позволяющей выбирать параметры регуляторов ПИД с использованием теории управления и математической модели квадрокоптера.

\section{Математическая молель}

Существует много научно-технических статей, посвященных разработке математической модели квадрокоптера [2-9]. Используя эти статьи, Динамическая модель для Parrot Ar Drone 2.0 с четырьмя винтами представлена в следующих формулах:

$$
\begin{aligned}
& I_{X X} \ddot{\phi}=\dot{\theta} \dot{\psi}\left(I_{y y}-I_{z z}\right)+J_{r} \dot{\theta} \Omega_{r}+l\left(-\Omega_{2}^{2}+\Omega_{4}^{2}\right) \\
& I_{Y Y} \ddot{\phi}=\dot{\phi} \psi\left(I_{z z}-I_{x x}\right)+J_{r} \dot{\theta} \Omega_{r}+l\left(-\Omega_{1}^{2}+\Omega_{3}^{2}\right) \\
& I_{z Z} \ddot{\psi}=\dot{\phi} \dot{\theta}\left(I_{x x}-I_{y y}\right)+J_{r} \dot{\Omega}_{r},
\end{aligned}
$$

Где:

- $\mathrm{I}_{x x}$ - момент инерции вокруг оси $\mathrm{x}$

- $\mathrm{I}_{y y}$ - момент инерции вокруг оси у

- $\mathrm{I}_{z z}$ - момент инерции вокруг оси z

- $\Omega_{1,} \Omega_{2}, \Omega_{3}, \Omega_{4}$-Угловые скорости роторов

- $\Omega_{r}$ - угловая скорость винта

Движения вокруг направлений $x$, у и z определяются уравнениями Ньютона-Эйлера. 


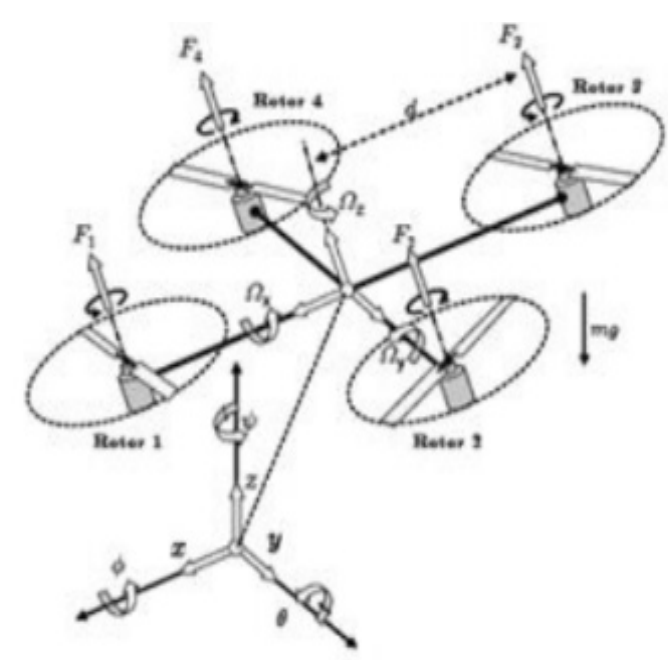

a)

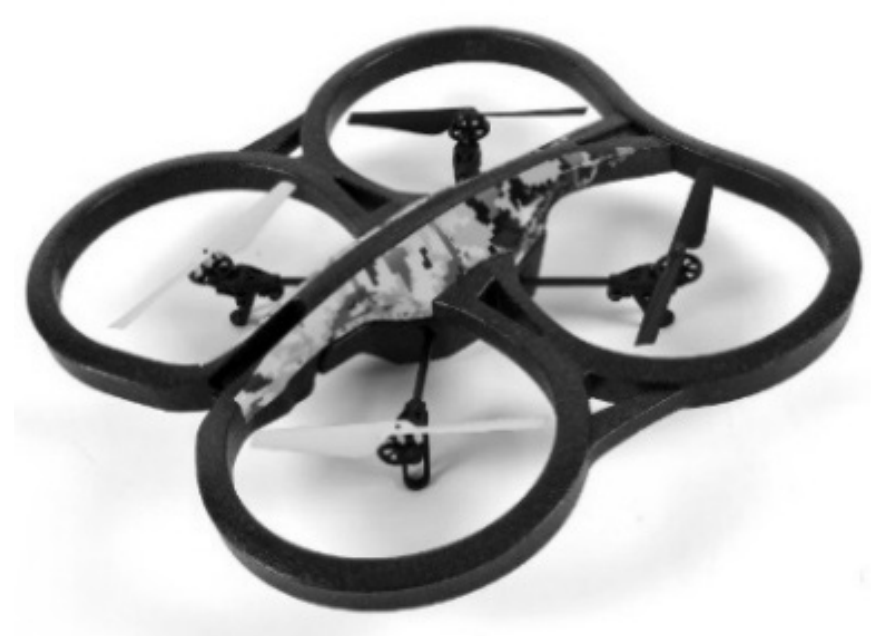

б)

Рис. 1. Геометрический облик квадрокоптера (а) и Parrot Ar. Drone 2.0 (б)

$$
\begin{aligned}
& m \ddot{z}=m g-(\cos \psi-\cos \phi) \sum_{i=1}^{4} \Omega_{i}^{2} \\
& m \ddot{x}=(\cos \phi \sin \theta \cos \psi+\sin \phi \sin \psi) \sum_{i=1}^{4} \Omega_{i}^{2} \\
& m \ddot{y}=(\cos \phi \sin \theta \sin \psi-\sin \phi \cos \psi) \sum_{i=1}^{4} \Omega_{i}^{2}
\end{aligned}
$$

Описанные уравнения (1) и (2) упрощаются путем добавления новых константов $\left(\mathrm{a}_{1}, \ldots, \mathrm{a}_{5}\right.$ и $\left.\mathrm{b}_{1}, \ldots, \mathrm{b}_{3}\right)$ и $\mathrm{U}_{1}, \ldots$, $\mathrm{U}_{4}$. Модель квадрокоптера модифицируется следующим образом [10].

$$
\dot{X}=f(X, U)=\left(\begin{array}{l}
\dot{\phi} \\
\dot{u} \dot{u} a_{1}+\dot{u} a_{2} \Psi_{r}+b_{1} U_{2} \\
\dot{u} \\
\dot{\phi} \dot{u} a_{3}+\dot{\phi} a_{4} \Psi_{r}+b_{2} U_{3} \\
\dot{u} \\
\dot{u} \dot{\phi} a_{5}+b_{3} U_{4} \\
\dot{z} \\
-g+(\cos \phi \cos u) \cdot(1 / m) \cdot U_{1} \\
\dot{x} \\
u_{x} \cdot(1 / m) \cdot U_{1} \\
\dot{y} \\
u_{y} \cdot(1 / m) \cdot U_{1}
\end{array}\right)
$$

где константы представлены следующими формулами:
$\begin{cases}a_{1}=\left(I_{Y Y}-I_{Z Z}\right) / I_{X X} & a_{5}=\left(I_{X X}-I_{Y Y}\right) / I_{Z Z} \\ a_{2}=-J_{r} / I_{X X} & b_{1}=l / I_{X X} \\ a_{3}=\left(I_{Z Z}-I_{X X}\right) / I_{Y Y} & b_{2}=l / I_{Y Y} \\ a_{4}=J_{r} / I_{Y Y} & b_{3}=l / I_{Z Z}\end{cases}$

И переменные со следующими формулами:

$$
\begin{aligned}
& \left\{\begin{array}{l}
U_{1}=b\left(\Omega_{1}^{2}+\Omega_{2}^{2}+\Omega_{3}^{2}+\Omega_{4}^{2}\right) \\
U_{2}=b\left(-\Omega_{2}^{2}+\Omega_{4}^{2}\right)
\end{array}\right. \\
& U_{3}=b\left(\Omega_{1}^{2}-\Omega_{3}^{2}\right) \\
& U_{4}=d\left(-\Omega_{1}^{2}+\Omega_{2}^{2}-\Omega_{3}^{2}+\Omega_{4}^{2}\right)
\end{aligned}
$$

Где b и d- коэффициенты движущей силы и силы реакции соответственно.

$$
\begin{aligned}
& u_{x}=(\cos \phi \sin \theta \cos \psi+\sin \phi \sin \psi) \\
& u_{y}=(\cos \phi \sin \theta \sin \psi-\sin \phi \cos \psi)
\end{aligned}
$$

Используя формулу (3), мы можем представить уравнение модели в виде матрицы, где векторы имеют вид:

$$
\begin{aligned}
& p=\left[\begin{array}{llllllllllll}
\phi & \dot{\phi} & \theta & \dot{\theta} & \psi & \dot{\psi} & z & \dot{z} & x & \dot{x} & y & \dot{y}
\end{array}\right]^{T} \\
& u=\left[\begin{array}{llll}
U_{1} & U_{2} & U_{3} & U_{4}
\end{array}\right]^{T}
\end{aligned}
$$


Таблица 1.

\begin{tabular}{|l|l|}
\hline Параметр & Величина \\
\hline$J_{x x}$ & 0.0086 кгм $^{2}$ \\
\hline$J_{y y}$ & $0.0086 \mathrm{KгM}^{2}$ \\
\hline$J_{z z}$ & $0.0172 \mathrm{KгM}^{2}$ \\
\hline$b$ & $3.13 \mathrm{e}^{-5}$ \\
\hline$d$ & $7.5 \mathrm{e}^{-7}$ \\
\hline$J r$ & $6 \mathrm{e}^{-5} \mathrm{~K}^{2}$ \\
\hline
\end{tabular}

Таким образом, мы получаем следующее матричное уравнение:

$$
\begin{aligned}
& \dot{X}=A p+B u \\
& y=C p+D u
\end{aligned}
$$

Матрицы А и В представлены следующим образом:

$$
A_{12 \times 12}=\left[\begin{array}{cccccccccccc}
0 & 1 & 0 & 0 & 0 & 0 & 0 & 0 & 0 & 0 & 0 & 0 \\
0 & 0 & 0 & \mathrm{a}_{24} & 0 & 0 & 0 & 0 & 0 & 0 & 0 & 0 \\
0 & \mathrm{a}_{32} & 0 & 1 & 0 & 0 & 0 & 0 & 0 & 0 & 0 & 0 \\
0 & 0 & 0 & 0 & 0 & 0 & 0 & 0 & 0 & 0 & 0 & 0 \\
0 & 0 & 0 & 0 & 0 & 1 & 0 & 0 & 0 & 0 & 0 & 0 \\
0 & 0 & 0 & 0 & 0 & 0 & 0 & 0 & 0 & 0 & 0 & 0 \\
0 & 0 & 0 & 0 & 0 & 0 & 0 & 1 & 0 & 0 & 0 & 0 \\
0 & 0 & 0 & 0 & 0 & 0 & 0 & 0 & 0 & 0 & 0 & 0 \\
0 & 0 & 0 & 0 & 0 & 0 & 0 & 0 & 0 & 1 & 0 & 0 \\
0 & 0 & 0 & 0 & 0 & 0 & 0 & 0 & 0 & 0 & 0 & 0 \\
0 & 0 & 0 & 0 & 0 & 0 & 0 & 0 & 0 & 0 & 0 & 1 \\
0 & 0 & 0 & 0 & 0 & 0 & 0 & 0 & 0 & 0 & 0 & 0
\end{array}\right], \text { n }
$$$$
B_{12 \times 4}=\left[\begin{array}{cccc}
0 & 0 & 0 & 0 \\
0 & b_{1} & 0 & 0 \\
0 & 0 & 0 & 0 \\
0 & 0 & b_{2} & 0 \\
0 & 0 & 0 & 0 \\
0 & 0 & 0 & b_{3} \\
0 & 0 & 0 & 0 \\
(\cos \phi \cos \theta) / m & 0 & 0 & 0 \\
0 & 0 & 0 & 0 \\
u_{x} / m & 0 & 0 & 0 \\
0 & 0 & 0 & 0 \\
u_{y} / m & 0 & 0 & 0
\end{array}\right]
$$

Где $a_{32}=\psi a_{3}+a_{4} \Omega_{r} \quad a_{24}=\psi a_{1}+a_{2} \Omega_{r}$. Соответственно $C$ и $D$ представлены следующим образом:

$$
C_{12 x 12}=\left[\left[\begin{array}{ccc}
1 & \cdots & 0 \\
\vdots & \ddots & \vdots \\
0 & \cdots & 1
\end{array}\right]\right] \text { и } D_{12 x 4}=\left[\left[\begin{array}{ccc}
1 & \cdots & 0 \\
\vdots & \ddots & \vdots \\
0 & \cdots & 1
\end{array}\right]\right]
$$

Уравнения для динамики Parrot Ar Drone 2.0 представлены в таблице 1.

\section{Система управления}

На рисунке 2 представлена системная блок-схема графической среды SIMULINK.

Для системного анализа необходимо учитывать несколько движений. В первом случае мы рассмотрели регулировку высоты четырьмя винтами. В этом случае входящий сигнал $\mathrm{U}_{l}$ был обработан, что необходимо для сохранения в заданном положении или для вертикального перемещения. В этом случае сигналы управления для всех двигателей одинаковы. Параметры ПИД регулятора следующие: $k_{p}=12, k_{i}=1,5$ и $k_{d}=6$. Окончательная передаточная функция по оси Z выглядит следующим образом:

$$
P I D_{z}(s)=k_{d} s+\frac{k_{i}}{s}+k_{p}=6 s+\frac{1.5}{s}+12
$$

На Рис. 3 показаны графики желаемых и моделированих переходов.

Во втором случае мы рассмотрим систему управления вращением вокруг оси Ү. Параметры для регулятора ПИД для этого направления: $k_{p}=0,1, k_{i}=0$ и $k_{d}=0,06$. Окончательная передаточная функция вокруг оси у выглядит следующим образом [11].

$$
P I D_{\text {yaw }}(s)=k_{d} s+k_{p}=0.06 s+0.1
$$

$\mathrm{PID}_{\text {yaw }}$ регулируется $\mathrm{U}_{4}$, что необходимо для поворота квадрокоптера вокруг объекта Ү. Чтобы проверить выбор параметров регулятора ПИД, мы наблюдали вращение вокруг оси Y и движение оси Z одновременно. Рис. 4. показывает желаемые и модулированные графики переходов. 


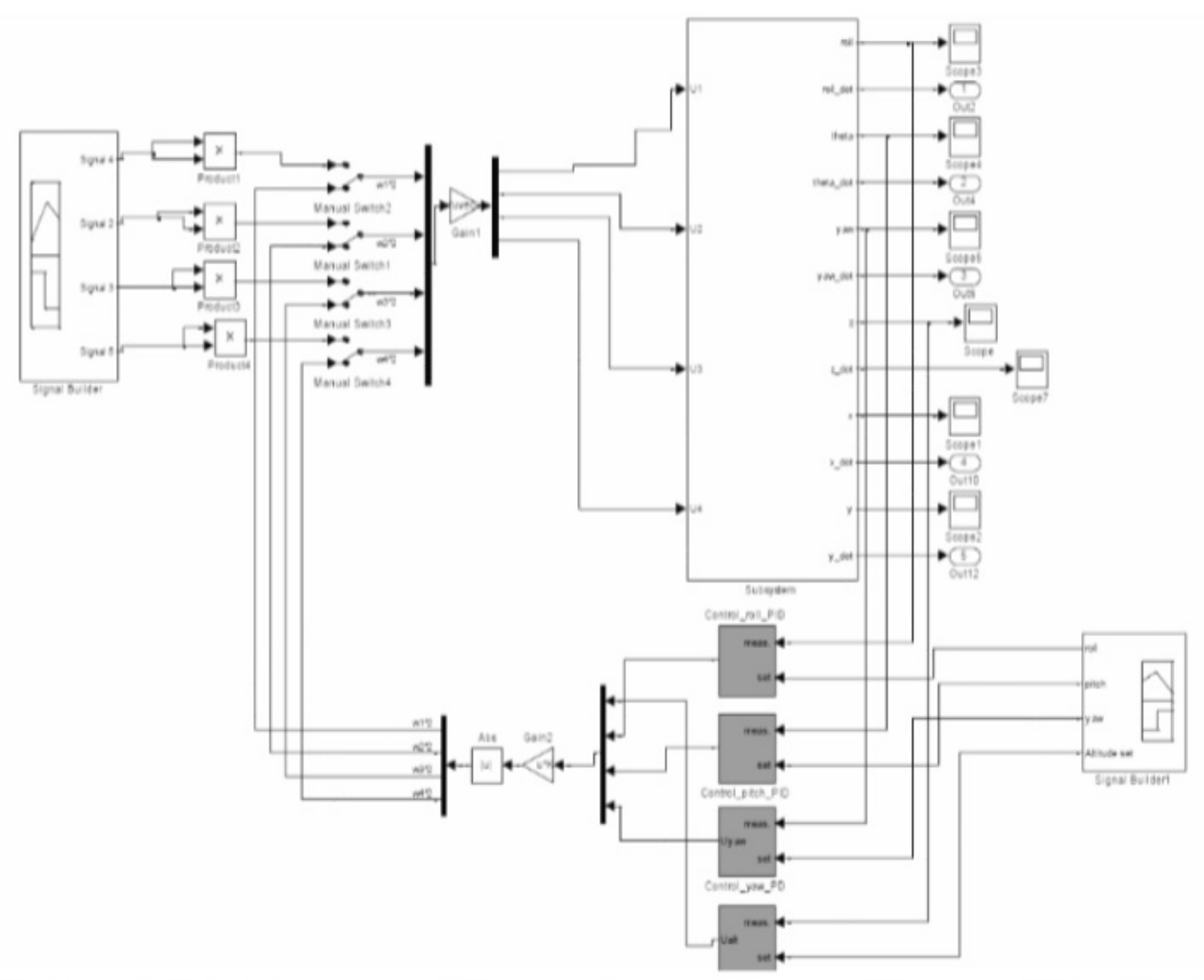

Рис. 2. Разработанная системная блок-схема

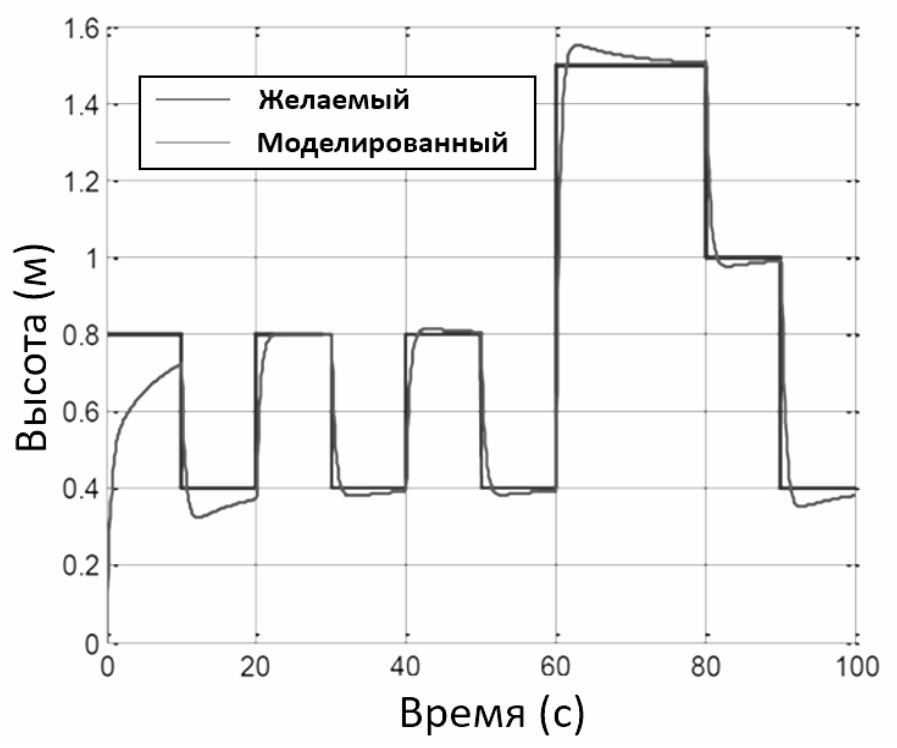

Рис. 3. График переходного движения по оси Z 

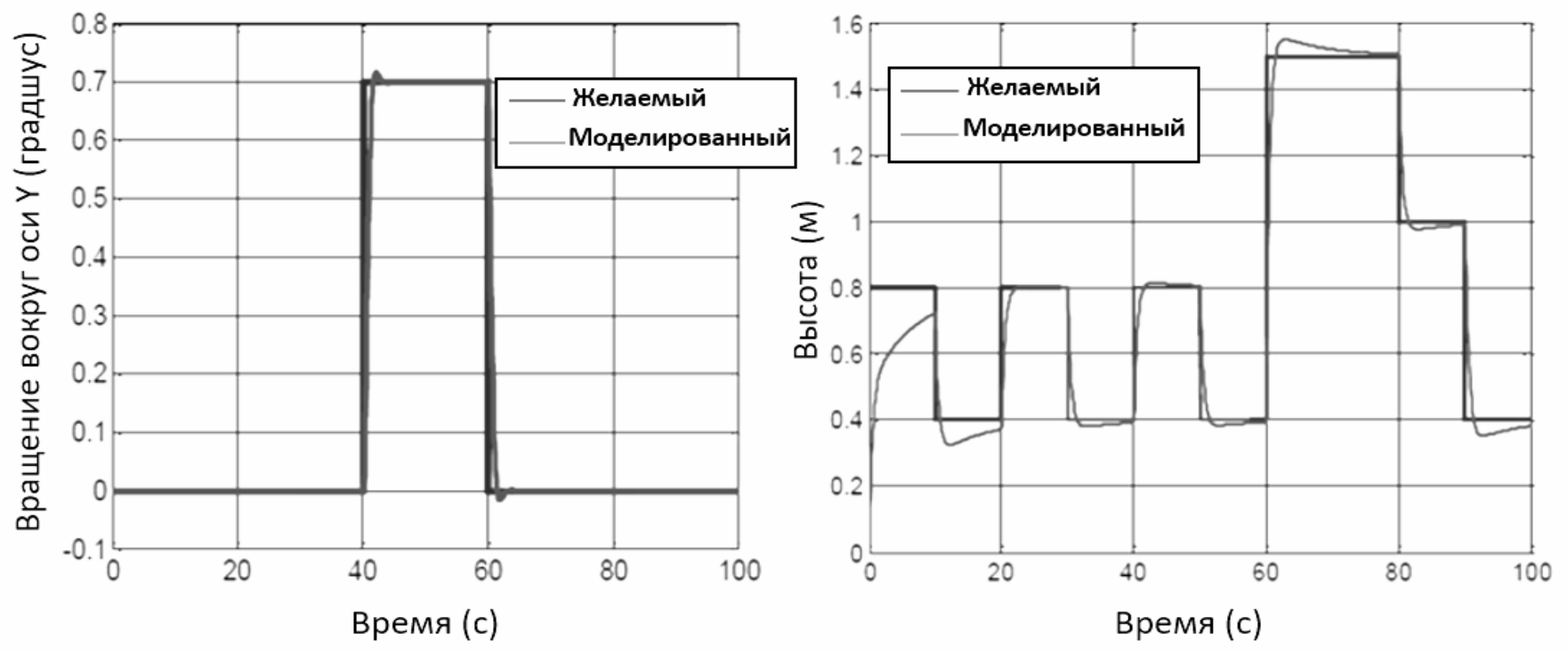

Рис. 4. Ү вращаться вокруг объекта одновременно графики переходного движения по оси Z
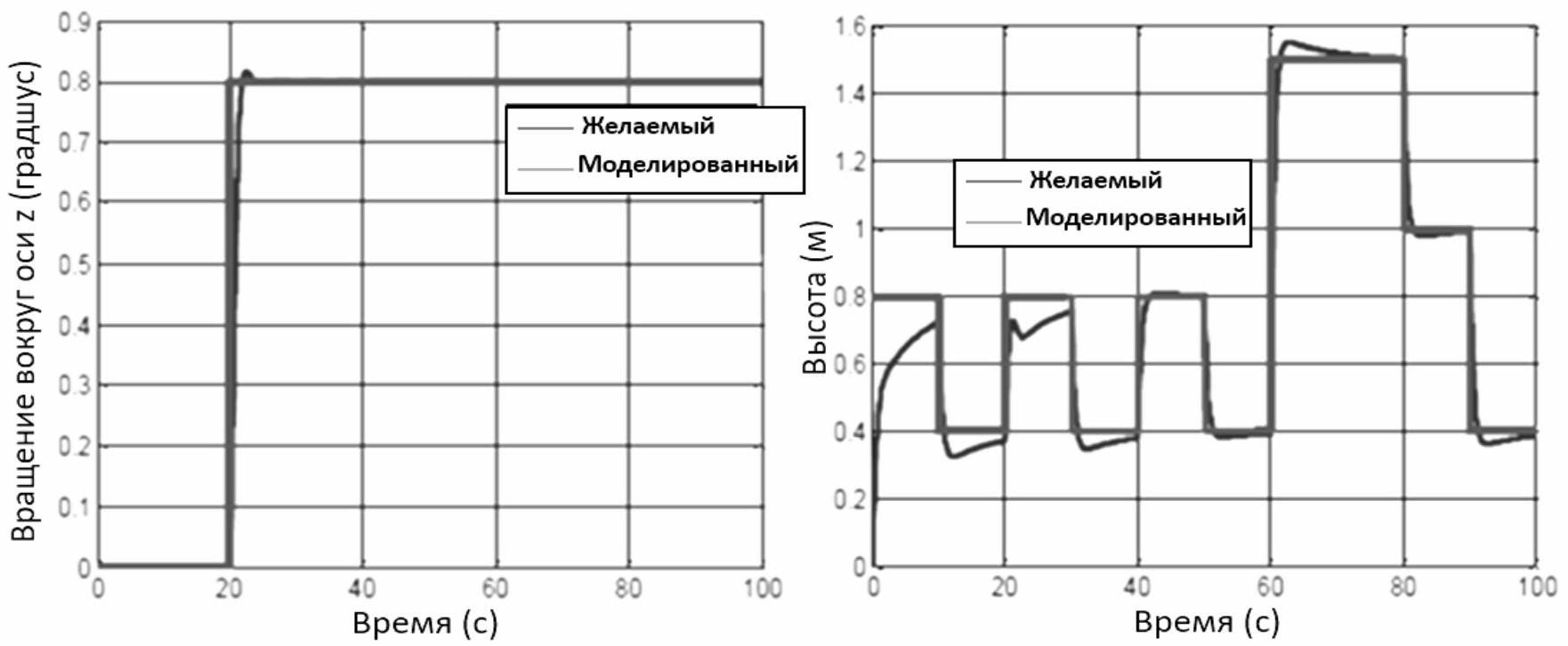

Рис. 5. X вращаться вокруг предмета и одновременно графики переходного движения по оси Z

В третьем случае рассматривается система управления вращением вокруг оси Z. Параметры для регулятора ПИД для этого направления: $k_{p}=0,3, k_{i}=0,01$ и $k_{d}=0,25$. Окончательная передаточная функция в повороте оси z выглядит следующим образом:

$$
P I D_{\text {pitch }}(s)=k_{d} s+k_{p}=0.15 s+0.2
$$

$\mathrm{PID}_{\text {pitch }}$ регулируется $U_{3}$, который необходим для перемещения четверки вперед и назад. На рисунке 5 показаны графики, полученные в процессе моделирования.

\section{Выво}

С помощью математической модели Parrot Ar. Drone 2.0 разработана система управления для программного обеспечения Matlab в графической среде SIMULINK и были выбраны параметры ПИД регуляторов. Предложенная система управления Parrot Ar. Drone 2.0, не чувствительна к изменениям движения. В последствии, мы можем установить полученные параметры регулятора ПИД в автопилоте Parrot Ar. Drone 2.0, чтобы исключить выбор и обеспечить стабильность системы во время тестирования, независимо от опыта оператора. 


\section{ЛИТЕРАТУРА}

1. McKerrow P. Modeling the Dragonflyer Four-Rotor Helicopter, International Conference on Robotics \& Automation, (2004), pp.3596-3601.

2. R. Lozano P. Castillo and A. Dzul. Stabilization of a mini rotorcraft with four rotors. IEEE Control Systems Magazine, pages 45-55, 2005.

3. Tayebi and S. McGilvray. Attitude stabilization of a four-rotor aerial robot. 43rd IEEE Conference on Decision and Control, pages 1216-1221, 2004.

4. R. Lozano. Unmanned Aerial Vehicles. Embedded Control, Published by ISTE Ltd, 2007.

5. S. Bouabdallah. Design and control of quadrotors with application to autonomous flying. PhD thesis, EPFL, 2007. 6.1, C.2

6. M. Claudia. Modelling and control of autonomous quad-rotor. Faculty of Engineering, Science and Medicine, University of Aalborg, Denmark, 2010:

7. P. I. Corke, Robotics, Vision \& Control: Fundamental Algorithms in MATLAB. Berlin: Springer-Verlag, 2011.

8. 0.N.Gasparyan, A. V. Davtyan, N. H. Nersisyan, A Control System Of A Quadcopter In The Simulink Software Environment Via Wi-Fi Signals, PROCEDINGS Of National Polytechnic University Of Armenia Information Technologies, Electronics, Radio Engineering, Yerevan 2019, №. 1, pp 21-29

9. Benallegue V. Mister and N. K. M'Sirdi. Exact linearization and non-interacting control of a 4 rotors helicopter via dynamic feedback. IEEE International Workshop on Robot and Human Interactive Communication, pages 586-593, 2001.

10. Derafa L., Madani T., Benalleque A. Dynamic Modeling and Experimental Identification of Four Rotors Helicopter Parameters, IEEE International Conference on Industrial Technology, (2006), pp.265-272.

11. Derafa L., Madani T., Ouladi A., Benallegue A. Four Rotors Helicopter Yaw Altitude Stabilization, Proceedings of the World Con-grass on Engineering, Vol. I, (2007), pp.104-110

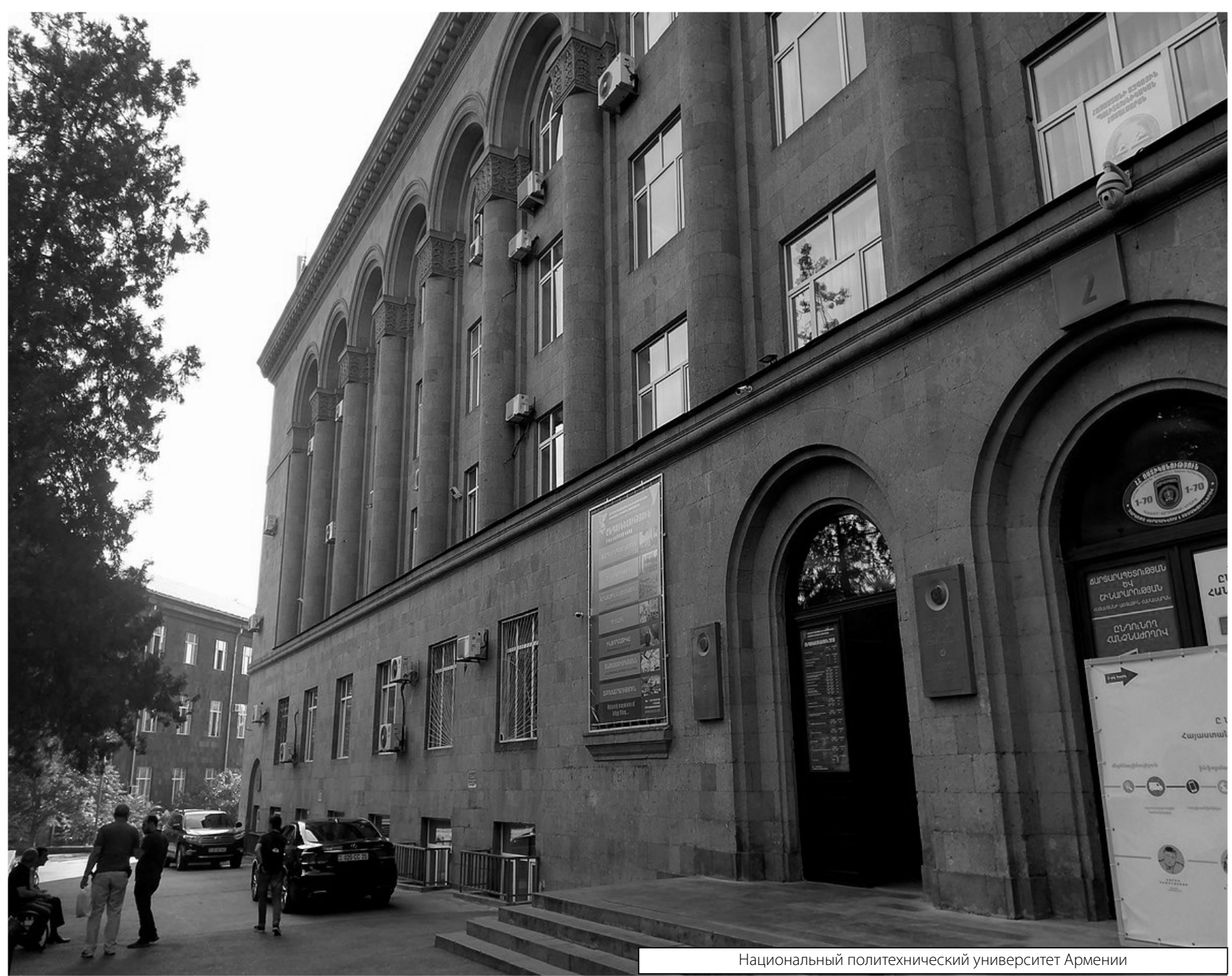

